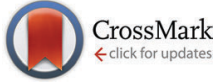

Cite this: Phys. Chem. Chem. Phys., 2016, 18, 14949

Received 28th August 2015, Accepted 25th February 2016

DOI: $10.1039 / \mathrm{c} 5 \mathrm{cp} 05132 \mathrm{k}$

www.rsc.org/pccp

\title{
Pair correlations that link the hydrophobic and Hofmeister effects $\dagger$
}

\author{
Quinn Alexander Besford,* Maoyuan Liu and Angus Gray-Weale*
}

\begin{abstract}
The Hofmeister effect describes how different ions make solutes more or less hydrophobic. The effect is thought to occur due to structural changes in the solvent induced by the ion's presence, particularly in water. In this study, the structural changes in water due to the presence of ions are investigated by molecular dynamics simulations of various monatomic ions in the SPC/E water model. Structural analyses reveal specific orientations of solvating waters around each of the ions studied. Using a new method, these orientations are quantified by a set of pair correlation functions that describe dipole-ion correlations in structure. These correlations are shown to contribute to the potential of mean force between waters and the ion of interest, and therefore to the free energy of the system. The magnitude of this free energy is found to result in a Hofmeister series for the various ions studied, therefore demonstrating a Hofmeister effect with respect to water's structure that is quantified by pair correlation functions. Most crucially, the pair correlations that lead to this Hofmeister effect also contribute to the hydrophobic effect (the entropy of hydrophobic solvation) [Liu et al., J. Chem. Phys., 2015, 142, 114117], and those which dominate the hydrophobic effect are modulated by an ion's presence, therefore demonstrating a mechanistic link between the two effects.
\end{abstract}

Cellular organisation and the biochemistry of an organism depend on water and dissolved ions. ${ }^{1-4}$ Different ions affect the conformations and activities of proteins, as well as protein solubility, crystallisation, and protein-protein interactions. ${ }^{5-8}$ The effects induced by ions in water are not limited to protein phenomena but also include the solubility of salts ${ }^{9}$ and hydrophobes, ${ }^{10}$ altered cloud points, ${ }^{11}$ altered reorientation dynamics of water in the first hydration shells, ${ }^{12,13}$ increased or reduced surface tensions, ${ }^{14-16}$ and changed molecular forces and colloidal stability. ${ }^{17}$ Furthermore, anions and cations have different affinities for the aqueous/air interface. ${ }^{18-20}$ The electric field around an ion causes the water molecules to re-arrange themselves, ${ }^{21}$ and it is thought that different ions exert their effects by changing the hydrogen bonding properties of water. ${ }^{22}$ This article investigates the perturbation of water's structure due to different monatomic ions by using a new method that accounts for pair correlations in structure. We recently used this method to account for the entropy of hydrophobic solvation. ${ }^{23}$ The aim here is to explore the contribution of pair correlations to the solvation free energy of ions in water, and whether a Hofmeister series can be found in these correlations.

The original Hofmeister series, proposed in 1888 by Franz Hofmeister, ${ }^{24}$ ordered various neutral salts on the minimal

School of Chemistry, The University of Melbourne, Melbourne, Australia.

E-mail: quinn.besford@gmail.com,gusgw@gusgw.net

$\dagger$ Electronic supplementary information (ESI) available: Details of calculations and RDF data. See DOI: $10.1039 / \mathrm{c} 5 \mathrm{cp} 05132 \mathrm{k}$ concentration required to precipitate a given protein from solution. ${ }^{25}$ This simple series showed that different ions make proteins more or less hydrophobic. The series has expanded since the time of Hofmeister to include more physical effects. ${ }^{8,17}$ The ordering of the series can change depending on the phenomena of interest, ${ }^{16,26}$ but broadly is classified into two extremes; kosmotropes and chaotropes. These terms are used to describe the capacity of a particular ion to "make" or "break" water structure, respectively, as this was thought to be central to the mechanism of the effect. ${ }^{15,27}$ However, the notion that the series exists due to changes in bulk water has not reached consensus and is still quite controversial. ${ }^{27,28}$ That water molecules in the first solvation shells of ions do not have the same average hydrogen bonding, in terms of extent, geometry, and dynamics as those in bulk water is beyond controversy. ${ }^{21}$ The situation for water molecules outside the first shell is less clear.

Diffraction measurements and molecular dynamics simulations have provided information on the structure of the solvation shells of ions. Näslund et al. ${ }^{29}$ reported X-ray absorption spectroscopy and X-ray Raman scattering data for ion solvation in water, finding that the hydrogen bonding network, in terms of forming and breaking, remains unchanged and only the waters closest to the ion are affected (i.e. the first solvation shell). This conclusion is supported by Funkner et $a .^{30}$ who found from a systematic terahertz absorption spectroscopy and molecular dynamics studies of a variety of divalent salts, that ion induced effects are confined to the first shell. Further complemented by femtosecond 
pump-probe spectroscopy measurements reported by Omta et al., ${ }^{31}$ who found the addition of ions had no influence on the rotational dynamics of water molecules outside the first solvation shell, suggesting the ions do not enhance or breakdown the hydrogen bond network in liquid water.

On the other hand, Mancinelli et al. ${ }^{32}$ interpreted neutron diffraction data from monovalent ionic solutions and found that the second solvation shell is perturbed from the bulk, indicating a larger range of an ion's perturbation of waters. This is supported by Tielrooij et $a .^{33}$ who used a coupled terahertz dielectric relaxation and femtosecond infrared spectroscopy to study water dynamics around different ions to conclude that in some cases the ion induced effects on water molecules extends well beyond the first solvation shell. A different approach by O'Brien $e t$ al. ${ }^{34}$ used infrared photodissociation spectroscopy to study the solvation patterns of $\mathrm{SO}_{4}{ }^{2-}\left(\mathrm{H}_{2} \mathrm{O}\right)_{n}$ clusters in the gas phase. O'Brien et al. found that for small clusters with $n<43$ the maximum of the $\mathrm{OH}$ vibrational band is blue-shifted from that of bulk water. That such a large number of water molecules show this pattern indicates ion-specific effects beyond the first solvation shell, ${ }^{35}$ since the first solvation shell contains about 12 water molecules. ${ }^{36}$ For larger clusters $n>43$ the broad frequency features increasingly resemble bulk water. This suggests that long-range structural effects beyond the first solvation shell can be observed. ${ }^{35}$ Some difficulty in interpreting structure past the ion may be compounded by the choice of the geometrics ${ }^{37}$ and the thermodynamics $^{38}$ of a hydrogen bond. More structural detail is needed in order to reconcile these different data on ion induced effects on water's network, and subsequent changes in hydrogen bonding.

Early models for the thermodynamics in aqueous systems generally treat the solvent as structureless. ${ }^{17}$ The Born theory of solvation $^{39}$ models interactions between the ion and solvent as purely electrostatic in origin with the ion viewed as a charged sphere of radius $r$ and the solvent as a dielectric continuum of dielectric constant $\varepsilon .{ }^{40}$ The free energy of transferring the ion from vacuum to the solvent is given by

$$
\Delta G_{\mathrm{Born}}=-\frac{q^{2}}{8 \pi \varepsilon_{0} r}\left(1-\frac{1}{\varepsilon}\right),
$$

where $q$ is the charge and $\varepsilon_{0}$ the permittivity of free space. Given its simplicity and neglect of other contributions, such as dispersion forces, this model is surprisingly accurate at reproducing the experimental solvation free energies. ${ }^{41}$ Some problems of the Born model are attributed to the use of ionic radii rather than cavity radii, ${ }^{40}$ and its use of a dielectric continuum model for the solvent; continuum models cannot take into account the disruptive nature of the ion on the structure of water. ${ }^{42}$ However, the model does highlight how large the contribution from purely electrostatic interactions are, where the Born model by itself has been shown by Rashin and Honig ${ }^{40}$ to reproduce the heats of solvation to within an error of less than $9 \%$. Other forces such as dispersion forces were neglected in early model calculations, probably because they were considered to be minor in comparison to Coulombic interactions. ${ }^{17}$

Dispersion forces between charged objects in an aqueous electrolyte system can be described by DLVO theory, ${ }^{43}$ which treats interparticle interactions in terms of a balance of attractive quantum mechanical treatment of van der Waals forces and repulsive electrical double-layer forces. ${ }^{8}$ The van der Waals forces are treated by Lifshitz theory, ${ }^{44}$ whereas the electrostatic forces are handled by a nonlinear Poisson-Boltzmann description. ${ }^{45}$ One of the major approximations in DLVO theory is the use of the Poisson-Boltzmann equation to describe the electrostatic interactions, as the method treats ions in solution as point-charges, thus losing the ion specificity, ${ }^{8}$ and therefore an account of the Hofmeister series. ${ }^{46}$ In addition, since van der Waals forces are intimately coupled to double layer electrostatic forces, ${ }^{47}$ more work is needed to improve the theory of dispersion forces in aqueous systems to reveal more detail on Hofmeister phenomena.

This article aims to show that structural changes induced by an ions presence in liquid water can be quantified by pair correlation functions that describe dipole-ion correlations in structure. These correlations ultimately contribute to the free energy of the system. In the following, simulations of various atomic ions in the SPC/E water model $^{48}$ are reported along a with rigorous structural analysis of waters around each ion. Pair correlation functions are derived for dipole-ion correlations and these will be shown to make a large contribution to the solvation free energy of the ions in water.

\section{Simulation method}

Classical molecular-dynamics (MD) simulations were performed using the SPC/E model of water. The Lennard-Jones potential parameters for SPC/E water used are the same as those given by Paschek $^{49}$ with $\sigma=(\mathrm{O}) 3.1656 \AA, \varepsilon k_{\mathrm{B}}{ }^{-1}(\mathrm{O})=78.2 \mathrm{~K}$, and $q(\mathrm{H})=$ $0.4238 e$. The SPC/E water model is chosen so as to compare results from these simulations to those reported by $\mathrm{us}^{23}$ for the entropy of hydrophobic solvation in SPC/E water. All ten ions were represented by a point charge with a Lennard-Jones (LJ). The non-polarisable potential parameters for water-ion interactions are the same as those used by Koneshan et al., ${ }^{50}$ and are summarised in Table S1 of the ESI. $\dagger$

The simulations were performed in the isobaric-isothermal $(N P T)$ ensemble with 512 water molecules and one ion. Simulations were performed using the Nosé-Hoover chain thermostat and barostat, ${ }^{51,52}$ with relaxation times of 1.5 and $2.4 \mathrm{ps}$, respectively. The electrostatic potentials were calculated using the P3M method on a grid of $16 \times 16 \times 16$ with an Ewald convergence parameter $\eta=0.18 .^{53}$ Bond constraints are handled using the SHAKE algorithm, ${ }^{54}$ and the integration performed using a timestep of 1 fs. Short-range interactions were cut-off at 18.5255 bohr. The simulations were equilibrated for $10 \mathrm{ps}$, and statistics collected over $1 \mathrm{~ns}$ trajectories. Statistics were sampled at 0.5 ps (500 MD steps). All simulations were performed over a range of temperatures from $260 \mathrm{~K}$ to $340 \mathrm{~K}$ at intervals of $10 \mathrm{~K}$, and $1 \mathrm{~atm}$ pressure.

\section{Results and discussion}

\subsection{Structure around ions}

The radial distribution function (RDF) between waters' oxygen and hydrogen centers with the $\mathrm{Ca}^{2+}$ ion are shown in Fig. 1A. 
A) 으ํ
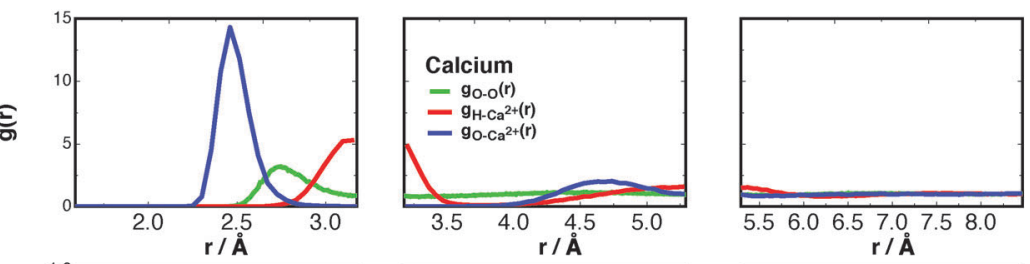

B)

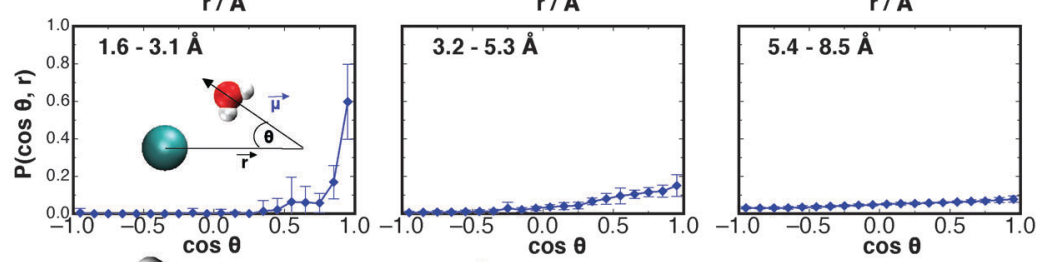

C)
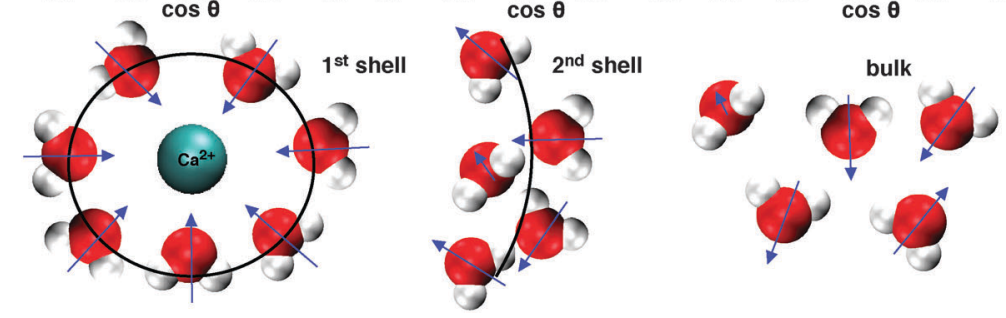

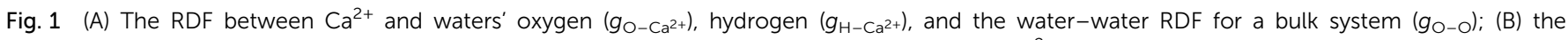
distribution of angles between waters' dipole moment vector and the displacement vector to the $\mathrm{Ca}^{2+}$ center, split over three sections with the distance from the ion given in the top left corner of each panel; (C) a schematic showing the distribution of waters (1st and 2nd solvation shell and bulk).

These are examined first so that the range of correlations in density may be later compared to ranges of dipole fluctuations.

The RDF for oxygen-ion correlations, $g_{\mathrm{O}-\mathrm{Ca}^{2+}}$, in the first solvation shell surrounding the $\mathrm{Ca}^{2+}$ shows the first solvation shell to be intensely populated with a peak maximum of about 14.31 at $2.46 \AA$ from the $\mathrm{Ca}^{2+}$ center, similar to the result obtained by Koneshan et al. ${ }^{50}$ For the hydrogen-ion correlation, $g_{\mathrm{H}-\mathrm{Ca}^{2+}}(r)$, the maximum is at $3.15 \AA$ with a height of 5.30 . Both functions decay to almost zero after the first solvation shell (see Table S2 in ESI $\dagger$ ), suggesting a tightly bound "crowd" of waters that do not leave the ion throughout the simulated trajectories. The second solvation shells for both functions are relatively small and both approach 1 at a distance of about $5.82 \AA$ from the $\mathrm{Ca}^{2+}$ center. For clarity, these functions are separated into three regions that roughly correspond to the first and second solvation shells, and bulk water for the $g_{\mathrm{O}_{-} \mathrm{Ca}^{2+}}(r)$ function. This is done so as to explore the distribution of dipole moment vectors, $\vec{\mu}$, around the $\mathrm{Ca}^{2+}$ ion. The distributions are calculated as the angle formed between the chemical dipole moment vector (+ve $\rightarrow-$ ve) of each water and the displacement vector, $\vec{r}$, to the ions center. The results are shown in Fig. $1 \mathrm{~B}$.

In the first solvation shell there is a strong orientation of waters' dipole moment vector directly towards the $\mathrm{Ca}^{2+}$ ion center. The orientational preference acts not only to shield the highly charged $\mathrm{Ca}^{2+}$ ion from surrounding waters, but also to stabilise the solvation shells around the ion, and therefore contribute to the free energy of solvation. We later show how this orientational preference leads to the lowering of the free energy of the surrounding waters. Interestingly, it can be seen that this orientation effect decays rapidly away from the first solvation shell. At the second solvation shell there is only a mild preference for an orientation towards the ion, and by $5.82 \AA$ outwards from the ion there is little preference for orientation, showing that the presence of the divalent ion does not significantly perturb the orientational structure of water past the second solvation shell into the bulk. Only the first solvation shell has a significant preference. These results are similar to those of Krekeler and Delle Site, ${ }^{55}$ who through Car-Parrinello MD of systems containing 32, 64, and 128 water molecules simulated for $5 \mathrm{ps}$, observed only the first solvation shell exhibits orientational effects. However, by simulating a larger system (512 water molecules) for a longer period of time (1 ns) we observe a mild orientational preference at the second solvation shell. Though other orientation effects, described by other correlation functions, may persist further into the bulk. These dipole moment orientation results are shown schematically in Fig. 1C.

The RDFs for $\mathrm{F}^{-}, \mathrm{Cl}^{-}, \mathrm{Br}^{-}, \mathrm{I}^{-}, \mathrm{Li}^{+}, \mathrm{Na}^{+}, \mathrm{K}^{+}, \mathrm{Rb}^{+}, \mathrm{Cs}^{+}$are shown in Fig. 2. The height of the first peak for both the anion and cation series follows a typical Hofmeister series; ${ }^{56}$ anions $\mathrm{F}^{-}>\mathrm{Cl}^{-}>\mathrm{Br}^{-}>\mathrm{I}^{-}$; cations $\mathrm{Ca}^{2+}>\mathrm{Li}^{+}>\mathrm{Na}^{+}, \mathrm{K}^{+}>\mathrm{Rb}^{+}>$ $\mathrm{Cs}^{+}$. These data show excellent agreement to other simulations reported by Lee and Rasaiah, ${ }^{57}$ giving confidence in the reproducibility of the simulated system. The smaller ions, most noticeably $\mathrm{F}^{-}, \mathrm{Li}^{+}$, and $\mathrm{Na}^{+}$, pull more waters into the first solvation shell than is usual for a water-water RDF. This may be viewed as a "structure-making" effect, but given that this effect is mainly limited to the 1st and only mildly to the 2 nd solvation shells of the $\mathrm{Ca}^{2+}$ ion (Fig. 1), the effect for the monovalent ions should be smaller. The solvation structure of the $\mathrm{K}^{+}$and $\mathrm{Rb}^{+}$ ions starts to resemble a population of waters that is usual for water-water correlations. The larger $\mathrm{Cs}^{+}$ion, and also the $\mathrm{Cl}^{-}$, $\mathrm{Br}^{-}$, and $\mathrm{I}^{-}$ions, occupy more space, therefore the first peak in the $g_{\mathrm{O}-\text { ion }}(r)$ for these ions occurs at a larger distance than the 

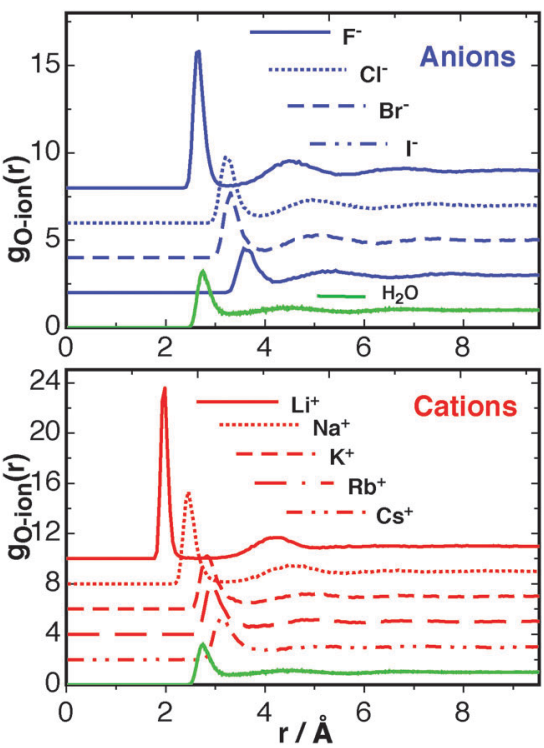

Fig. 2 The radial distribution functions (RDFs) between waters' oxygen and the ion center for all the monovalent ions studied, given at $300 \mathrm{~K}$ and $1 \mathrm{~atm}$ in the NPT ensemble. The green dash-dotted lines correspond to the usual water-water RDF. The RDFs are shifted vertically for clarity $\left(\mathrm{F}^{-}:+8\right.$, $\left.\mathrm{Cl}^{-}:+6, \mathrm{Br}^{-}:+4, \mathrm{I}^{-}:+2, \mathrm{Li}^{+}:+10, \mathrm{Na}^{+}:+8, \mathrm{~K}^{+}:+6, \mathrm{Rb}^{+}:+4, \mathrm{Cs}^{+}:+2\right)$.

water-water $g(r)$. With respect to the free energy of solvation, the larger ions occupy more space that would otherwise be occupied by water, therefore creating a destabilising cavity contribution to the free energy. This is explored further later in this article The RDF results are summarised in Table S2 of the ESI, $\dagger$ with the distance at the first peak in the RDF along with the corresponding minima, and the same for the second solvation shell shown. The first minima in all the O-ion RDFs are much smaller than the first minimum of the water-water $\mathrm{RDF}$, revealing a relatively tight binding of the first solvation shell for all ions studied.

The distribution of orientations of waters for each of the monovalent ions are shown in Fig. 3. The distributions are averaged over only the first solvation shell for each ion. The lower and upper limits for the averaging is the start of the first

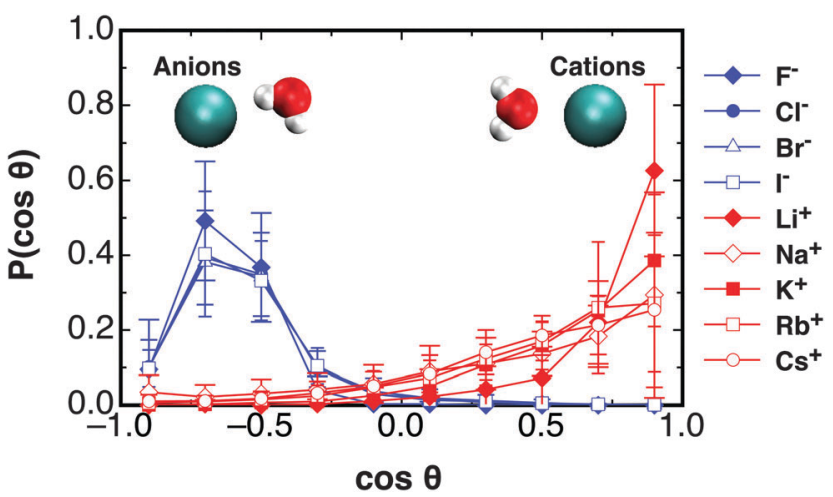

Fig. 3 The distribution of angles between waters' dipole moment vector and the displacement to the ion for all the monovalent ions studied at the first peak in each of their RDFs, as given in Table S1 in ESI, $\uparrow$ given at $300 \mathrm{~K}$ and $1 \mathrm{~atm}$ in the NPT ensemble. peak in the RDF for each ion, and the distance to the first minima, as given in Table S2 (ESI $\dagger$ ). Similar to the case for $\mathrm{Ca}^{2+}$, it is seen that the cations cause the hydrating waters to preferentially orientate their dipole vector directly at the ion. The strongest orientational constraint is found for the $\mathrm{Li}^{+}$ion, and this eases into a broader distribution of angles in travelling down the group 1 series to $\mathrm{Cs}^{+}$. The anions have a more defined distribution at $\cos \theta \approx-0.7$, indicating the first hydrating waters orientate themselves to point one hydrogen atom at the ion. This is similar for all the halides. In the following an analysis of how far these orientational preferences perturb water correlations out towards the bulk is given.

\subsection{Dipole-ion correlations}

As shown above in the structural analyses of water around ions, there is a definite change in structure of the first solvation shell of an ion. We show here that the restricted orientations of water's dipole moments around each ion occurs so as to lower the free energy of the system and stabilise the solvation shells. This stabilisation may be quantified by pair correlation functions that contribute to the potential of mean force between each water and the ion, and to the free energy. Recently, $\mathrm{we}^{23}$ reported derivations for water-water and water-solute pair correlation functions, and found that these correlations can explain the entropy of hydrophobic solvation of small non-polar species in water. This was similar to results obtained by Lazaridis and Paulaitus ${ }^{58}$ who found that the entropy and heat capacities of hydrophobic hydration are well accounted for by solute-water correlations alone. In the following the water-water and water-solute correlation functions are applied to the simulated aqueous systems.

Water-ion correlations as a function of separation $r$, may be written in terms of a potential of mean force $\psi(r)$ (see Section 3 of Liu et al. ${ }^{23}$ for derivations), given as

$$
\psi(r)=-k_{\mathrm{B}} T \frac{1}{2\left(g_{\alpha \mathrm{w}}(r)\right)^{2}} \sum_{n=1}^{\infty}\left(h_{n 0 n}(r)\right)^{2},
$$

where $\alpha$ and w denote an ion and water, respectively, $g_{\alpha w}(r)$ is the RDF between them, $k_{\mathrm{B}}$ is Boltzmann's constant, $T$ the absolute temperature, and $h_{n 0 n}$ is an expansion coefficient describing preferential orientations of dipole moments (see $\mathrm{ESI} \dagger$ for the first 9 functions). Following the treatment of free energy changes given by Hansen and McDonald (see especially Sections $3.4,3.5$, and $\left.4.3^{59}\right)$, the contribution of $\psi(r)$ to the free energy of the fluid may be written

$$
G_{\psi}=4 \pi \rho \int_{0}^{\infty} \mathrm{d} r r^{2} \psi(r) g_{\alpha \mathrm{w}}(r),
$$

where $\rho$ is the number density of species. The RDF between an ion and a water may be written as

$$
g_{\alpha \mathrm{w}}(r)=\frac{\left\langle N_{\alpha \mathrm{w}}(r)\right\rangle_{\mathrm{f}}}{\rho_{\alpha} \rho_{\mathrm{w}}\left(4 \pi r^{2} \Delta r+\frac{\pi}{3} \Delta r^{3}\right) V} \approx \frac{\left\langle N_{\alpha \mathrm{w}}(r)\right\rangle_{\mathrm{f}}}{N_{\alpha} \rho_{\mathrm{w}} 4 \pi r^{2} \Delta r},
$$

where $V$ is the volume, $N_{\alpha \mathrm{w}}(r)$ the number of pairs of $\alpha-\mathrm{w}$ at a separation of $r, N_{\alpha}$ the number of species $\alpha$, and the angle 
brackets $\langle\cdot\rangle_{\mathrm{f}}$ indicate an ensemble average over frames. It is now convenient to simplify eqn (3) to allow rapid analysis across simulations. Substitution of eqn (4) into eqn (3), along with simplification leads to

$$
\begin{aligned}
\frac{\Delta G_{\psi}}{N_{\alpha}} & =\rho_{\mathrm{w}} \sum_{i} 4 \pi r_{i}^{2} g_{\alpha \mathrm{w}}(r) \psi_{\alpha \mathrm{w}}\left(r_{i}\right) \Delta r, \\
& =\sum_{i} \frac{\left\langle N_{\alpha \mathrm{w}}\left(r_{i}\right)\right\rangle_{\mathrm{f}}}{N_{\alpha}} \psi_{\alpha \mathrm{w}}\left(r_{i}\right),
\end{aligned}
$$

and insertion of eqn (2) into eqn (6), and allowing $h_{n 0 n}(r)=$ $\left\langle\Phi_{n 0 n}(r)\right\rangle$, the final expression is obtained

$$
\frac{\Delta G_{\psi}}{N_{\alpha}}=-\frac{k_{\mathrm{B}} T}{2 N_{\alpha}} \sum_{i} \frac{\sum_{n}\left\langle\sum \Phi_{n 0 n}\left(r_{i}\right)\right\rangle_{\mathrm{f}}^{2}}{\left\langle N_{\alpha \mathrm{W}}\left(r_{i}\right)\right\rangle_{\mathrm{f}}} .
$$

Eqn (7) can be readily evaluated by binning waters at a distance $r$ from the ion of interest and counting the angles formed between the dipole moment vector and its displacement from the ion, and then calculating their contribution to the $\Phi_{n 0 n}$ basis functions, and ultimately to $\Delta G_{\psi}$. The different basis functions that contribute to $\psi(r)$, and ultimately to $\Delta G_{\psi}$, for each of the ions studied are shown in the ESI. $\dagger$

The contribution of $\Delta G_{\psi}$ to the free energy of solvation for each of the ions is compared in Fig. 4 . Fig. 4 shows that a large stabilising free energy exists due to the correlation of waters' dipole moments with each ion, for both the anions and cations. The free energy has a weak temperature dependence, where the free energy becomes more negative with increasing temperature. The magnitude of $\Delta G_{\psi}$ is about an order of magnitude lower than the experimental free energy of solvation, ${ }^{60}$ which is to be expected as great as the total free energy of solvation is dominated by direct Coulombic interactions (calculated through the simple Born model in eqn (1)). It is seen here that a significant contribution to the free energy of solvation arises purely from correlations between dipole moment orientations and ions in solution.

The lower plot of Fig. 4 shows $\Delta G_{\psi}$ for the $\mathrm{Ca}^{2+}$ ion in SPC/E water, where a much larger contribution from the divalent ion is seen, about an order of magnitude greater than that for the $\mathrm{Cs}^{+}$ion. At room temperature the stabilisation due to $\psi(r)$ for calcium is of the order of $-170 \mathrm{~kJ} \mathrm{~mol}^{-1}$, about $-165 \mathrm{~kJ} \mathrm{~mol}^{-1}$ greater than $\psi(r)$ for the solvation of methane. ${ }^{23}$ The increase in the magnitude of $\psi(r)$ for the calcium ion hints at a simple scaling relationship stemming from the charge.

Most intriguing is that the sequence of stabilisation due to $\psi(r)$ in going from most stabilising to least stabilising follows the typical Hofmeister series. Fig. 4 shows specific ion effects where the smallest ions stabilise the solvating waters to the greatest extent, such as the $\mathrm{F}^{-}, \mathrm{Ca}^{2+}$ and $\mathrm{Li}^{+}$, whereas the larger ions do this to a lesser degree, especially for $\mathrm{I}^{-}$and $\mathrm{Cs}^{+}$, indicating a more hydrophobic like character of these larger ions. This Hofmeister series follows the one given by Kunz ${ }^{56}$ exactly, however there is not one unique Hofmeister series as the series changes order depending on the property of interest, such as surface tensions or protein denaturing. The order of
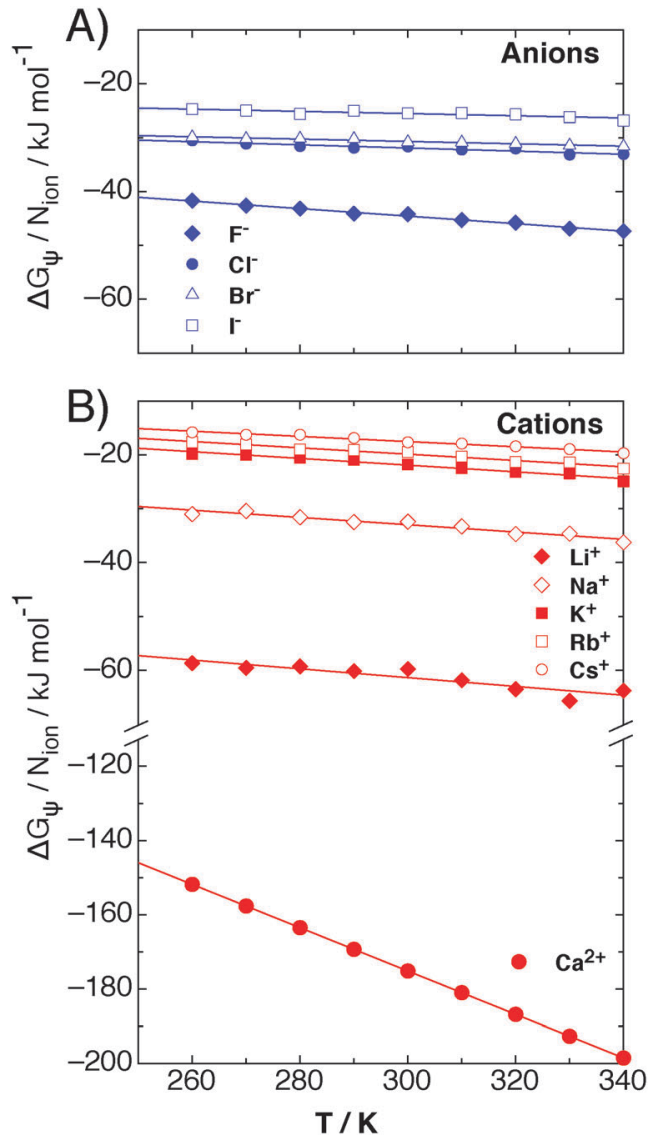

Fig. 4 The contribution of dipole-ion correlations, $\psi(r)$, to the free energy of solvation, denoted by $\Delta G_{\psi}$, for (A) anions and (B) cations, as calculated from eqn (7), for each of the atomic ions in SPC/E water at $1 \mathrm{~atm}$ in the NPT ensemble. The lines are fits to eqn (8) for each ion.

the series found in $\psi(r)$, with respect to the magnitude of $-\Delta G_{\psi}$, follows

Anions

$$
\mathrm{F}^{-}>\mathrm{Cl}^{-}>\mathrm{Br}^{-}>\mathrm{I}^{-}
$$

Cations

$$
\mathrm{Ca}^{2+}>\mathrm{Li}^{+}>\mathrm{Na}^{+}>\mathrm{K}^{+}>\mathrm{Rb}^{+}>\mathrm{Cs}^{+}
$$

where the contribution of $\psi(r)$ for the monovalent ions decrease in travelling down their respective groups. The ions commonly referred to as kosmotropes (structure makers) are situated on the left side of the anion series, whereas the ions commonly referred to as chaotropes (structure breakers) are situated on the left hand side of the cation series. Given that the $\psi(r)$ interaction causes a change in structure around an ion, and that $\Delta G_{\psi}$ produces a Hofmeister series, the mechanism of $\psi(r)$ is an explanation of why there is an ordering of these ions in a Hofmeister series due to their structure making or breaking capabilities. This observation and conclusion is based on the simulated equilibrated trajectories of waters' dipole moments around an ion in solution. Fig. 5 shows a schematic of how the solvating waters are orientated for four example ions. Note that the $\mathrm{F}^{-}$ion most strongly structures the solvating waters on the 

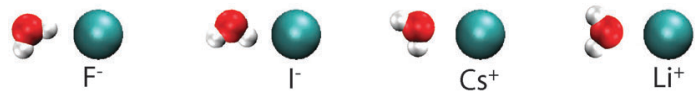

Fig. 5 A cartoon illustrating how the solvating waters are orientated around the fluoride, iodide, cesium and lithium ions.

left hand side of Fig. 5, whereas the $\mathrm{Ca}^{2+}$ and $\mathrm{Li}^{+}$ions most strongly structure the solvating waters on the right hand side of Fig. 5, with respect to the magnitude of $\Delta G_{\psi}$.

The right hand side of the series begins to approach a similar $\Delta G_{\psi}$ as that which is found from correlations between waters' dipole moments and small hydrophobic molecules. For example, in other work we found ${ }^{23}$ that $\Delta G_{\psi}$ for xenon at $300 \mathrm{~K}$ is about $-7 \mathrm{~kJ} \mathrm{~mol}^{-1}$, whereas that for cesium is about $-18 \mathrm{~kJ} \mathrm{~mol}^{-1}$. This demonstrates that the larger ions do become more hydrophobic with increasing ionic radius, and that this analysis is consistent with previous work. ${ }^{23}$

The lines in Fig. 4 are fits to the form, ${ }^{61}$

$$
\Delta G=\Delta C_{\mathrm{p}}\left(T-T_{\mathrm{h}}-T \log \frac{T}{T_{\mathrm{s}}}\right),
$$

allowing the solvation entropy $\Delta S_{\psi}$ to be obtained via differentiation $\left(\Delta S_{\psi}=-\left(\frac{\partial \Delta G_{\psi}}{\partial T}\right)_{\mathrm{P}}\right)$. The solvation entropies, due to $\psi(r)$ are shown in Table 1 for each of the ions at $300 \mathrm{~K}$ and $1 \mathrm{~atm}$, along with the free energy contributions from $\psi(r)$. It can be seen in Table 1 that the entropic component of $\psi(r)$ is positive for all ions, however this is small compared to the total entropy of solvation.

The changes in structure due to $\psi(r)$ are large and may therefore disrupt water-water correlations. Recently, $\mathrm{we}^{23}$ derived an asymptotic expression for water-water correlations that is denoted $\phi(r)$ to distinguish it from water-solute correlations $(\psi(r))$. The form of $\phi$ between two thermally rotating dipole moments, in SPC/E water, was found to be

$$
\begin{aligned}
& \phi(r)=-k_{\mathrm{B}} T \frac{27 g_{\mathrm{K}}{ }^{2}(\varepsilon-1)^{2}}{16 \pi^{2}(1+2 \varepsilon)^{2} \rho^{2}} \frac{1}{r^{6}}, \\
& =-k_{\mathrm{B}} T \frac{27 g_{\mathrm{K}}^{2}}{64 \pi^{2}}\left[1+O\left(\frac{1}{\varepsilon}\right)\right] \frac{1}{\rho^{2} r^{6}},
\end{aligned}
$$

where $\varepsilon$ is the static permittivity and $g_{\mathrm{K}}$ is the Kirkwood $g$-factor,

Table 1 A comparison of dipole-ion correlations $\left(\Delta G_{\psi}\right)$ contributions to the free energy and entropy of solvation of ions in water, at $300 \mathrm{~K}$

\begin{tabular}{lcc}
\hline Ions & $\Delta G_{\psi} / \mathrm{kJ} \mathrm{mol}^{-1}$ & $\Delta S_{\psi} / k_{\mathrm{B}}$ \\
\hline $\mathrm{F}^{-}$ & -44.2 & 8.4 \\
$\mathrm{Cl}^{-}$ & -31.7 & 3.5 \\
$\mathrm{Br}^{-}$ & -30.8 & 2.6 \\
$\mathrm{I}^{-}$ & -25.5 & 2.5 \\
& & \\
$\mathrm{Ca}^{2+}$ & -175.21 & 19.4 \\
$\mathrm{Li}^{+}$ & -59.7 & 9.9 \\
$\mathrm{Na}^{+}$ & -32.4 & 8.2 \\
$\mathrm{~K}^{+}$ & -21.8 & 7.6 \\
$\mathrm{Rb}^{+}$ & -19.4 & 7.1 \\
$\mathrm{Cs}^{+}$ & -17.6 & 5.9
\end{tabular}

which describes local orientational correlations between dipole moments. $g_{\mathrm{K}}$ for SPC/E water is about 2.5. ${ }^{23}$ As with $\psi(r)$, the free energy due to $\phi(r)$ may be estimated as a sum over pairs, where the most convenient form to analyse simulations is via

$$
G_{\phi}=\left\langle\sum_{i<j} \phi\left(r_{i j}\right)\right\rangle_{\mathrm{f}} .
$$

The contribution of $\phi(r)$ to the solvation free energy is simply the difference in eqn (11) for simulations with and without an ion, denoted $\Delta G_{\phi}$. To check that the stabilisation from $\psi(r)$ exceeds the decrement in $\phi(r)$ due to the ions presence, the magnitude of the decrement in $\phi(r)$ is compared in Fig. 6. The dielectric properties required for this calculation are given in Fig. S4 of the ESI. $\dagger$

Fig. 6 shows that each ion causes a positive change in free energy due to their disrupting effect on $\phi(r)$ between waters. The magnitudes of these positive changes in free energy are greater for the larger more hydrophobic atoms but is still less than the negative stabilising free energy due to $\psi(r)$. The decrement in $\Delta G_{\phi}$ for the monovalent ions is significantly lower than the stabilisation due to $\psi(r)$ by some $20-50 \mathrm{~kJ} \mathrm{~mol}^{-1}$, and about $170 \mathrm{~kJ} \mathrm{~mol}^{-1}$ for calcium, meaning that the stabilisation due to $\psi(r)$ represents a large, stabilising contribution to the free energy of solvation that exceeds dipolar correlations that are lost due to the ions presence.

The different correlation functions, $\psi(r)$ and $\phi(r)$, are compared for the fluorine ion in water at $300 \mathrm{~K}$ in Fig. 7. The asymptotic $\phi(r)$
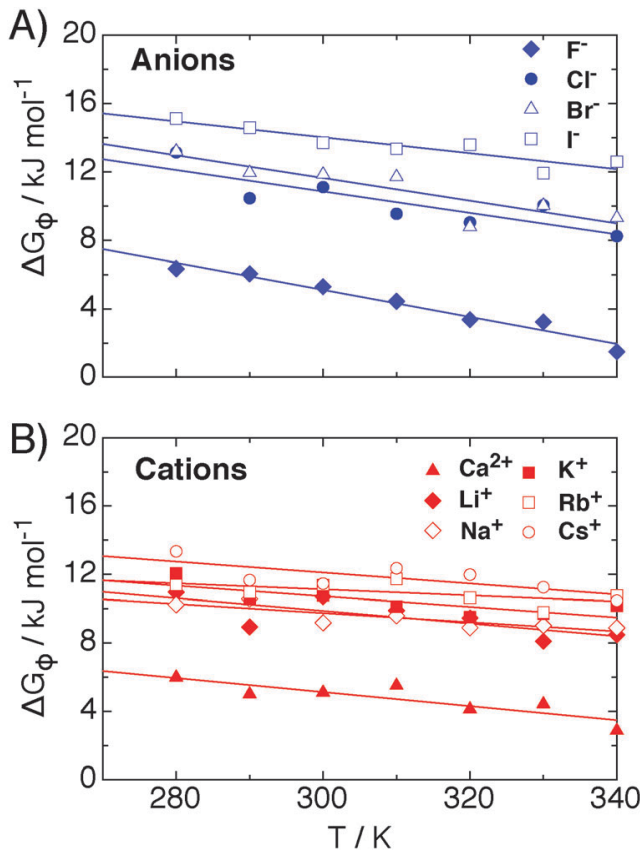

Fig. 6 The contribution of $\phi(r)$ to the free energy of solvation for each of the (A) anions and (B) cations studied, in SPC/E water at $1 \mathrm{~atm}$ in the NPT ensemble. The change in free energy due to $\phi$ is calculated by difference of eqn (11) for pure water and ionic simulations. The lines are linear fits to each data set, and the dielectric properties for the bulk system are given in Fig. S4 of the ESI. $\dagger$ 


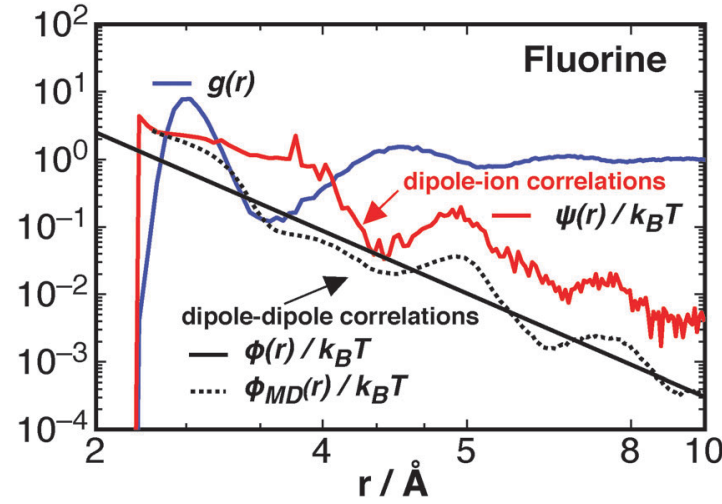

Fig. 7 A comparison of the radial distribution function between waters oxygen center and the fluorine ion (blue line), the dipole-ion correlations from $\psi(r)$ (red line), and the asymptotic expression for $\phi(r)$ (solid black line), and the full expression obtained from simulation, $\phi_{\mathrm{MD}}(r)$ (black dotted line). All calculated from simulations at $300 \mathrm{~K}$ and $1 \mathrm{~atm}$ of the NPT ensemble. Note this is a log-log plot.

(eqn (10)), solid black line, is shown with the full expression $\phi_{\mathrm{MD}}(r)$, black dotted line, which is calculated from simulated trajectories of pure water (see Section 3 of Liu $e t a l^{23}$ ), as

$$
\phi_{\mathrm{MD}}(r)=-k_{\mathrm{B}} T \sum_{l_{1}} \sum_{l_{2}} \sum_{l \geq\left|l_{1}-l_{2}\right|}^{l_{1}+l_{2}}\left(h_{l_{1} l_{2} l}^{\mathrm{ww}}(r)\right)^{2} / 2\left(g^{\mathrm{ww}}(r)\right)^{2},
$$

where the allowed basis sets of the expansion coefficient $h_{l_{1} l_{2} l}$ are given by Liu et al., ${ }^{23}$ and the superscripts ww denotes a function performed on pairs of waters.
The dipole-ion correlations follow the same qualitative longrange behaviour as $\phi_{\mathrm{MD}}(r)$, at least within the $12 \AA$ studied, where the second and third peaks between $\phi_{\mathrm{MD}}(r)$ and $\psi(r)$ roughly line up, though the $\psi(r)$ contribution is about an order of magnitude greater. This result shows that the ion's presence is felt at a longer range than the first solvation shell. This is not due to hydrogen bonding, the quantity commonly checked through experiment, but is due to correlations between rotations that contribute to potential of mean force between water molecules and the ion. These ion-water interactions are greater than the $\phi(r)$ interactions between waters and thus represents a significant change in solution thermodynamics due to the ions presence. At short ranges the stabilisation due to $\psi$ decays approximately as $r^{-2}$, the same as what is expected for a fixed dipole (or constrained)-charge pair potential. ${ }^{62}$

For a closer analysis of $\phi(r)$ between waters in these systems, $\phi(r)$ was calculated between waters where at least one water molecule is within the first and also the second solvation shell of the ion. These results are shown in Fig. 8, along with the $\phi(r)$ for the entire system, a reference $\phi(r)$ for a bulk system, and also the $\psi(r)$ functions, for each of the extreme anionic and cationic monovalent ions.

Fig. 8 shows features that are specific to each ion type. The fluoride ion causes an enhancement of $\phi(r)$ between waters just at the first solvation shell, while the second solvation shell $\phi(r)$ is almost the same as the bulk system. $\psi(r)$ for this system is greater than all other components of the dipole correlation free energy for almost the entirety of the distance up to $r_{\text {cut }}$. The iodine ion causes a similar effect to that caused by fluorine, but to a lesser extent. For lithium, the waters in the first solvation
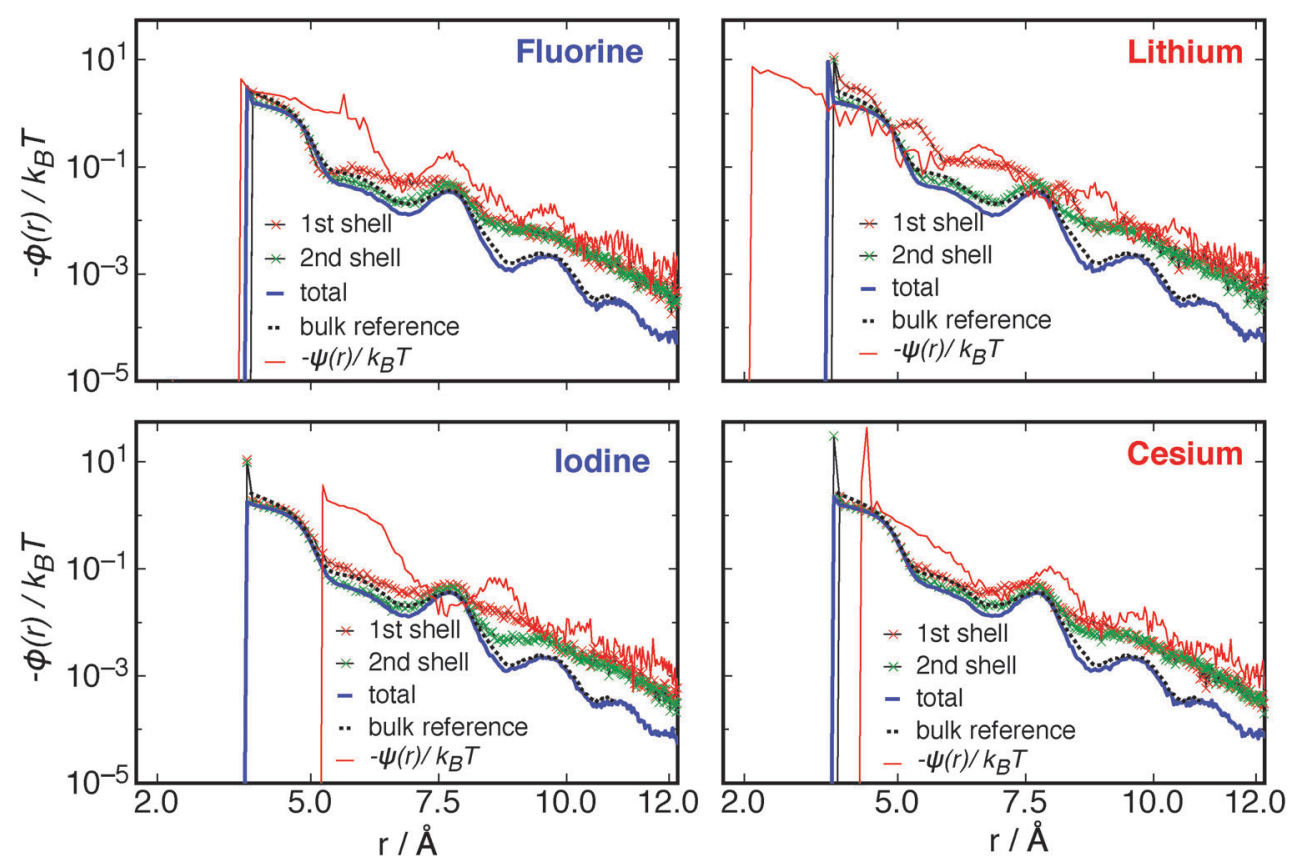

Fig. 8 A closer comparison of the enhancement and decrement of $\phi(r)$ for waters in the first (red crossed lines) and second (green crossed lines) solvation shells around the ion, and that in the bulk reference (black dotted line) and the total $\phi(r)$ for the ion system (blue line). Also shown are the $\psi(r)$ functions for each ion (red lines). The two extreme ions are shown for both the anionic and cationic monovalent ions studied, at $300 \mathrm{~K}$ and 1 atm. Note these are log-log plots. 
shell have a substantially increased correlation with other waters, where $\phi(r)$ even exceeds $\psi(r)$ in some places along the correlation curve. The cesium ion, on the other hand, causes very little enhancement of water-water correlations around the ion. Even though there are enhancements of water-water correlations in the solvation shells around the ions, the overall contribution to the systems free energy is small, where there are only few waters in each shell thus averaging over all waters in the system still results in a system size decrement in $\phi(r)$ between waters. This can be seen as the blue curves in Fig. 8 in comparison to the bulk reference (black dotted lines).

The data in Fig. 8 reveal a complex modulation of water-water interactions depending on the distance from the ion. This may contribute to the observed inconsistencies between different experiments on hydrogen bonding as a function of distance from the ion (see introduction). However, the orientational correlations observed in Fig. 8 are long-ranged interactions that are distinct from hydrogen bonding and as such does not indicate if hydrogen bonding has changed.

As has been shown by Liu et al., ${ }^{23}$ the $\phi(r)$ interaction is responsible for the hydrophobic effect. These data in Fig. 8 reveal a new mechanism for the direct influence of ions on hydrophobic interactions.

2.2.1 Solvation energies including electrostatic and dispersion contributions. The main contributions to the solvation free energy $\Delta G_{\text {solv }}$ stem from electrostatic effects that result in solvent immobilisation, electrostriction, and dielectric saturation of the solvation shells. ${ }^{60}$ As has been shown in the previous section, the $\psi(r)$ interaction is long-ranged and produces a significant contribution to the $\Delta G_{\text {solv }}$, the calculation of which is based on the explicit structure as determined from MD simulations. Other models have been proposed that account for dispersion interactions that are missed by the Born model of solvation, which only accounts for electrostatic effects.

Duignan et al. ${ }^{41,63}$ developed a continuum model of dispersion contributions to $\Delta G_{\text {solv }}$, based on macroscopic quantum electrodynamics, similar to Lifshitz theory, ${ }^{64}$ using frequency dependent multipole polarisabilities of molecules to predict the dipole, quadruple, and octupole contributions. This dispersion model predicts a negative (favourable) solvation free energy, which is offset by a positive cavity contribution stemming from the region of lost water-water interactions due to the ions presence. This cavity contribution is calculated as the free energy of expanding a surface in water, given as

$$
\Delta G_{\mathrm{c}}=\sigma_{\mathrm{ion}} 4 \pi R_{\mathrm{s}}{ }^{2}
$$

where $\sigma_{\text {ion }}$ is the macroscopic surface tension of water, and $R_{\mathrm{s}}$ is the distance to the first peak in the ion-oxygen RDF. The strongest dispersion contribution is that from dipolar interactions, calculated from

$$
\Delta G_{\mathrm{D}}=-\frac{6 k_{\mathrm{B}} T}{R_{\mathrm{cav}} 3} \sum_{n=0}^{\infty}\left(1-\frac{1}{2} \delta_{n_{0}}\right) \alpha\left(i \zeta_{n}\right) \frac{\varepsilon\left(i \zeta_{n}\right)-1}{2 \varepsilon\left(i \zeta_{n}\right)+1}
$$

where $\alpha_{1}\left(i \zeta_{n}\right)$ is the dipolar polarisability tensor, $\varepsilon\left(i \zeta_{n}\right)$ the dielectric function, and $R_{\text {cav }}$ is an adjusted solvation radius, found from $R_{\text {cav }}=R_{\mathrm{S}}-0.84 \AA$. The model, like the Born model (eqn (1)), is sensitive to this choice, as noted by the authors. This radius is different from that used to calculate the cavity contribution, where $R_{\text {cav }}$ represents the region where water is not significantly polarised, and $R_{\mathrm{s}}$ gives the average distance between the centers on the solute and the water molecules in the first solvation shell. As a continuum model, interactions resulting from correlations in structure are neglected and therefore components of the free energy that result from long-ranged interactions are missed, such as those due to $\psi(r)$ interactions. However, when the model is combined with the Born model the combined model accurately reproduces the solvation energy of the ions studied with the chosen parameters for $R_{\text {cav }}$ and $R_{\mathrm{s}}$. For a similar comparison, assuming force additivity, here the free energy resulting from $\psi(r)$ and $\phi(r)$ interactions, $\Delta G_{\psi, \phi}$, is added onto the electrostatic contribution, $\Delta G_{\text {elec }}$, calculated through the Born model (eqn (1)). This is shown and compared to Duignan et al.'s model and to experimental solvation free energies ${ }^{65,66}$ for the monovalent ions in Fig. 9. The electrostatic contribution used is that calculated through the Born model by Duignan et al., ${ }^{41}$ and is summarised in ESI. $\dagger$ For this comparison the electrostatic contribution is assumed to be independent of $\Delta G_{\psi, \phi}$.

Fig. 9 shows that in combination with the Born electrostatic solvation energy, the free energy resulting from dipole-ion and dipole-dipole correlations $\left(\Delta G_{\psi, \phi}\right)$ gives good agreement with experimental solvation free energies. This total theoretical solvation free energy from $\Delta G_{\psi, \phi}$ is close to that predicted for the cavity, dispersion, and electrostatic contributions, $\Delta G_{\mathrm{c}, \mathrm{D}}$ and $\Delta G_{\text {Born }}$, respectively. Duignan et al.'s ${ }^{41}$ model for the $\Delta G_{\mathrm{c}, \mathrm{D}}$ contributions, when combined with $\Delta G_{\text {Born }}$, better captures the solvation free energy for the $\mathrm{Na}^{+}$and $\mathrm{Li}^{+}$ions in water. The deviation for these two ions may stem from the accuracy of the potentials used in the simulations, and that of the SPC/E water model. To better understand these deviations a more rigorous comparison to computational solvation free energies is

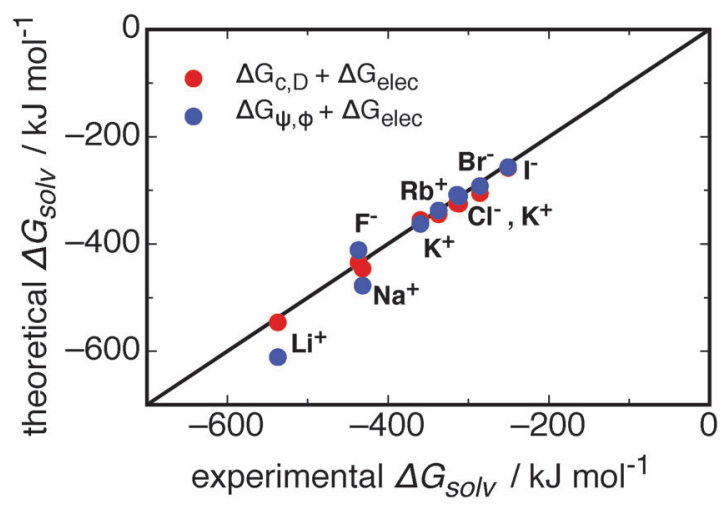

Fig. 9 A comparison of different models for the contributions to the solvation free energies of monatomic ions. The contribution of $\psi(r)$ and $\phi(r)$ from this work, $\Delta G_{\psi, \phi}$, is combined with the electrostatic contribution from the Born model (data given in ESI $\dagger$ ), $\Delta G_{\mathrm{Born}}{ }^{41}$ and shown as blue circles. The model of Duignan et al. ${ }^{41}$ for the cavity and dispersion, $\Delta G_{\mathrm{C}, \mathrm{D}}$, and electrostatic contributions are shown as red circles. The line is for $x=y$. Note that $\Delta G_{\psi, \phi}$ is calculated from simulations. 
Table 2 A comparison of dipole-dipole correlations $\left(\Delta G_{\phi}\right)$ and dipoleion correlations $\left(\Delta G_{\psi}\right)$ to Duignan et al.'s ${ }^{63}$ model of dipolar dispersion $\Delta G_{D}$ and cavity costs $\Delta G_{c}$ for the solvation of ions. Free energies are given in units of $\mathrm{kJ} \mathrm{mol}^{-1}$, and the free energies resulting from $\psi$ and $\phi$ are given at $300 \mathrm{~K}$ and $1 \mathrm{~atm}$ in the NPT ensemble

\begin{tabular}{lrllllr}
\hline Ions & $\Delta G_{\phi}$ & $\Delta G_{\psi}$ & $\Delta G_{\mathrm{c}}$ & \multicolumn{1}{c}{$\Delta G_{\mathrm{D}}$} & $\Delta G_{\psi, \phi}$ & $\Delta G_{\mathrm{c}, \mathrm{D}}$ \\
\hline $\mathrm{F}^{-}$ & 5.3 & -44.2 & 39.1 & -100.5 & -38.9 & -61.4 \\
$\mathrm{Cl}^{-}$ & 11.1 & -31.7 & 55.8 & -90.3 & -20.5 & -34.5 \\
$\mathrm{Br}^{-}$ & 11.9 & -30.8 & 61.1 & -93.3 & -19.0 & -32.2 \\
$\mathrm{I}^{-}$ & 13.7 & -25.5 & 72.2 & -85.4 & -11.81 & -13.2 \\
& & & & & & \\
$\mathrm{Li}^{+}$ & 10.7 & -59.7 & 23.1 & -7.1 & -49.1 & 16.0 \\
$\mathrm{Na}^{+}$ & 9.2 & -32.4 & 30.1 & -21.5 & -23.2 & 8.6 \\
$\mathrm{~K}^{+}$ & 10.8 & -21.8 & 42.4 & -46.2 & -11.0 & -3.8 \\
$\mathrm{Rb}^{+}$ & 11.4 & -19.4 & 46.5 & -61.6 & -8.0 & -15.1 \\
$\mathrm{Cs}^{+}$ & 11.5 & -17.6 & 52.7 & -74.9 & -6.1 & -22.2 \\
& & & & & &
\end{tabular}

required, which is outside the scope of our work. It is important to note that the free energies resulting from $\psi(r)$ and $\phi(r)$ interactions stem from parameter free calculations on explicit simulated trajectories, and that when combined with the Born electrostatic free energy these quantities give good agreement with experiment.

In order to better compare the different contributions to the solvation free energy, and ultimately to clarify specific ions effects between the models, the components of Duignan et al.' $\mathrm{s}^{41}$ model are compared to that stemming from $\psi(r)$ and $\phi(r)$ in Table 2.

Table 2 reveals interesting features in comparing the cavity and dispersion model to $\Delta G_{\psi}$ and $\Delta G_{\phi}$. The total free energy for the anions between each theory is of a similar magnitude, following the same trend in decreasing down the halide group. The cations, on the other hand, do not show this same agreement. The trend for $\Delta G_{\psi, \phi}$ is the same as that for the anions, whereby the magnitude of the solvation free energy decreases in going down the group 1 metals. This can be understood with regards to the smaller cations most strongly structuring the solvating waters and therefore producing a larger stabilisation due to ion-dipole correlations. The $\Delta G_{\mathrm{c}, \mathrm{D}}$ contribution for these cations follows the opposite trend, where the $\mathrm{Li}^{+}$ion gives a positive solvation free energy, reducing down to a small negative contribution for $\mathrm{K}^{+}$, and a reasonably large negative contribution for $\mathrm{Cs}^{+}$. This latter trend is counter-intuitive, as the small $\mathrm{Li}^{+}$ion might be expected to produce a similar solvation free energy to the $\mathrm{F}^{-}$ion due to the similar structural effects induced by its presence (see Fig. 2), and the $\mathrm{Cs}^{+}$ion may be expected to give a free energy similar to a small hydrophobe, given its large size and low influence on structure (compare Fig. 2 and 3 , for example). It is this difference for the cations that allows the cavity and dispersion model to better predict the experimental solvation free energy for the $\mathrm{Li}^{+}$and $\mathrm{Na}^{+}$ions.

However, the important point of this comparison is that there is a large contribution to the solvation free energy, similar in magnitude to that from Duignan et al. ${ }^{41}$ model, that is found in correlations in structure $\left(\Delta G_{\psi, \phi}\right)$. This cannot be found with the assumption of a structureless dielectric continuum, as implied by eqn (14). The ion-dipole correlations studied here demonstrate the long-ranged interaction between waters and an ion, and it is this long-ranged interaction between molecular species that breaks the assumption of a structureless dielectric continuum for aqueous water (see Discussion on p. 338 of Landau and Lifshitz ${ }^{67}$ ). By taking the explicit structure of water around an ion into account, new contributions to the free energy of the system have been found, and these contributions produce a Hofmeister series between the ions studied.

Most importantly, the mechanism that leads to our Hofmeister series $(\psi(r))$ is the same as that which leads to the entropy of hydrophobic solvation $(\phi(r)){ }^{23}$ In addition, the $\phi(r)$ interaction is modulated by the presence of ions (Fig. 8). This demonstrates a link between the two effects. This link has not previously been established, to our best knowledge, but various experiments have hinted at a close relationship between the two effects. Such as experiments that have shown the addition of salt reduces the solubility of nonpolar solutes, such as benzene, in water ${ }^{22,68}$ in roughly the same rank-ordering and at the same concentrations as those found for protein precipitations. ${ }^{25,69,70}$ This relationship is not limited to solubility either, where Geisler et al. ${ }^{71}$ reported atomic force microscopy measurements of hydrophobic and Hofmeister effects on the adhesion of spider silk proteins onto a solid substrate. They find the desorption forces follow the Hofmeister series for anions and is influenced by the hydrophobicity of the silk protein, indicating the hydrophobic and Hofmeister effects are closely related. This is evidenced further by chemical reactions, where Rizzo ${ }^{72}$ found a Hofmeister series when different ions are present in the hydrophobically accelerated Diels-Alder reaction.

The theory behind $\psi(r)$, that produces a Hofmeister series, is linked to the hydrophobic effect ${ }^{23}$ through the contribution of rotational correlations between dipoles and ions to the potential of mean force between species (see eqn (2) above and eqn (15) of Liu et al. ${ }^{23}$ ). This represents a general mechanism leading to specific ion effects that links in with hydrophobic phenomena. The calculations performed in this article for $\psi(r)$ and $\phi(r)$ interactions all are based on the simulated explicit trajectories of water molecules surrounding each ion of interest. The only free parameter for these calculations is the pair potential used in the MD simulation. The results shown, based on interactions arising from dipole-ion and dipoledipole correlations, therefore prove a mechanistic link between hydrophobic and Hofmeister effects.

\section{Conclusions}

The structural changes induced by ions in water have been investigated by MD simulations of various monatomic ions in SPC/E water, with radial distribution functions and orientational distributions reported. Strong ion-water correlation functions were found, the strength of which varies between different ions. The range of ion-water correlations was found to extend far past the first and second solvation shells around ions, demonstrating that an ion's presence is "felt" at large distances, upwards of $12 \AA$, from the ion's center. These correlation functions contribute to a stabilising free energy, the magnitude of which spans $-17.6 \mathrm{~kJ} \mathrm{~mol}^{-1}$ for 
cesium to $-175.2 \mathrm{~kJ} \mathrm{~mol}^{-1}$ for calcium. This stabilisation was found to far exceed the decrement in water-water interactions due to the ion's presence, demonstrating a real gain in solvation free energy from ion-water correlations.

The main finding in this article is that when the ions are ordered with respect to the magnitude of this new free energy, a Hofmeister series emerges. The Hofmeister series ranks different ions on their ability to make solutes more or less hydrophobic (the Hofmeister effect). In comparing to other models for the solvation free energy of ions in water, the computational method presented here gives similar agreement to experimental solvation free energies. The results presented stem directly from pair correlation functions between thermally rotating dipole moments and each ion in solution, and the contribution of this correlation to the total free energy of the system. Most crucially, the pair correlations that lead to this Hofmeister effect also contribute to the hydrophobic effect, ${ }^{23}$ and those which dominate the hydrophobic effect are modulated by an ion's presence. This article has therefore demonstrated a molecular coupling between the Hofmeister and hydrophobic effects.

\section{Acknowledgements}

This research was supported by a grant from the Australian Research Council (Grant No. DP110103388), and by a grant of computer time from the Victorian Life Sciences Computation Initiative (Grant No. VR0252). The Australian Government is acknowledged for Australian Postgraduate Awards (QAB and $\mathrm{ML}$ ). QAB gratefully acknowledges the University of Melbourne for receipt of the Albert Shimmins Award.

\section{References}

1 M. Chaplin, Nat. Rev. Mol. Cell Biol., 2006, 7, 861-866.

2 P. Lo Nostro and B. W. Ninham, Chem. Rev., 2012, 112, 2286-2322.

3 J. A. Rupley, E. Gratton and G. Careri, Trends Biochem. Sci., 1983, 8, 18-22.

4 K. D. Collins, Biophys. Chem., 2006, 119, 271-281.

5 B. Hribar, N. T. Southall, V. Vlachy and K. A. Dill, J. Am. Chem. Soc., 2002, 124, 12302-12311.

6 K. A. Dill, Biochemistry, 1990, 29, 7133-7155.

7 D. K. Eggers and J. S. Valentine, J. Mol. Biol., 2001, 314, 911-922.

8 Y. Zhang and P. S. Cremer, Curr. Opin. Chem. Biol., 2006, 10, 658-663.

9 N. Peruzzi, B. W. Ninham, P. Lo Nostro and P. Baglioni, J. Phys. Chem. B, 2012, 116, 14398-14405.

10 L. M. Pegram and M. T. Record, J. Phys. Chem. B, 2008, 112, 9428-9436.

11 H. Schott and A. E. Royce, J. Pharm. Sci., 1984, 73, 793-799. 12 J. Boisson, G. Stirnemann, D. Laage and J. T. Hynes, Phys. Chem. Chem. Phys., 2011, 13, 19895.
13 D. Laage, G. Stirnemann, F. Sterpone, R. Rey and J. T. Hynes, Annu. Rev. Phys. Chem., 2011, 62, 395-416.

14 L. M. Pegram and M. T. Record, J. Phys. Chem. B, 2007, 111, 5411-5417.

15 W. J. Xie and Y. Q. Gao, J. Phys. Chem. Lett., 2013, 4, 4247-4252.

16 N. Schwierz, D. Horinek and R. R. Netz, Langmuir, 2010, 26, 7370-7379.

17 W. Kunz, P. Lo Nostro and B. Ninham, Curr. Opin. Colloid Interface Sci., 2004, 9, 1-18.

18 L. Yang, Y. Fan and Y. Q. Gao, J. Phys. Chem. B, 2011, 115, 12456-12465.

19 D. Horinek and R. R. Netz, Phys. Rev. Lett., 2007, 99, 226104.

20 R. R. Netz and D. Horinek, Annu. Rev. Phys. Chem., 2012, 63, 401-418.

21 Y. Marcus, Chem. Rev., 2009, 109, 1346-1370.

22 R. L. Baldwin, Biophys. J., 1996, 71, 2056-2063.

23 M. Liu, Q. A. Besford, T. Mulvaney and A. Gray-Weale, J. Chem. Phys., 2015, 142, 114117.

24 F. Hofmeister, Arch. Exp. Pathol. Pharmakol., 1888, 24, 1-30.

25 M. Cacace, E. Landau and J. Ramsden, Q. Rev. Biophys., 1994, 30, 241-277.

26 D. F. Parsons, M. Boström, T. J. Maceina, A. Salis and B. W. Ninham, Langmuir, 2010, 26, 3323-3328.

27 Y. Zhang, S. Furyk, D. E. Bergbreiter and P. S. Cremer, J. Am. Chem. Soc., 2005, 127, 14505-14510.

28 R. Zangi, J. Phys. Chem. B, 2010, 114, 643-650.

29 L.-A. Näslund, D. C. Edwards, P. Wernet, U. Bergmann, H. Ogasawara, L. G. M. Pettersson, S. Myneni and A. Nilsson, J. Phys. Chem. A, 2005, 109, 5995-6002.

30 S. Funkner, G. Niehues, D. A. Schmidt, M. Heyden, G. Schwaab, K. M. Callahan, D. J. Tobias and M. Havenith, J. Am. Chem. Soc., 2012, 134, 1030-1035.

31 A. W. Omta, M. F. Kropman and S. Woutersen, Science, 2003, 301, 347-349.

32 R. Mancinelli, A. Botti, F. Bruni, M. A. Ricci and A. K. Soper, Phys. Chem. Chem. Phys., 2007, 9, 2959-2967.

33 K. J. Tielrooij, N. Garcia-Araez, M. Bonn and H. J. Bakker, Science, 2010, 328, 1006-1009.

34 J. T. O'Brien, J. S. Prell, M. F. Bush and E. R. Williams, J. Am. Chem. Soc., 2010, 132, 8248-8249.

35 D. Paschek and R. Ludwig, Angew. Chem., 2011, 50, 352-353.

36 X. B. Wang, X. Yang, J. B. Nicholas and L. S. Wang, Science, 2001, 294, 1322-1325.

37 R. Kumar, J. R. Schmidt and J. L. Skinner, J. Chem. Phys., 2007, 126, 204107.

38 K. A. T. Silverstein, A. D. J. Haymet and K. A. Dill, J. Am. Chem. Soc., 2000, 112, 8037-8041.

39 M. Born, Z. Phys., 1920, 1, 4548.

40 A. Rashin and B. Honig, J. Phys. Chem., 1985, 59, 5588-5593.

41 T. T. Duignan, D. F. Parsons and B. W. Ninham, J. Phys. Chem. B, 2013, 117, 9421-9429.

42 W. R. Fawcett, J. Phys. Chem. B, 1999, 103, 11181-11185. 
43 E. Verwey and J. T. Overbeek, Theory of the stability of lyophobic colloids: the interaction of sol particles having an electric double layer, Elsevier, New York, 1948.

44 I. Dzyaloshinskii, E. Lifshitz and L. Pitaevskii, Sov. Phys.-Usp., 1961, 73, 153-176.

45 M. Boström, D. Williams and B. Ninham, Phys. Rev. Lett., 2001, 87, 168103.

46 B. W. Ninham, Adv. Colloid Interface Sci., 1999, 83, 1-17.

47 B. W. Ninham and V. Yaminsky, Langmuir, 1997, 13, 2097-2108.

48 H. J. C. Berendsen, J. R. Grigera and T. P. Straatsma, J. Phys. Chem., 1987, 91, 6269-6271.

49 D. Paschek, J. Chem. Phys., 2004, 120, 6674-6690.

50 S. Koneshan, J. C. Rasaiah and S. H. Lee, J. Phys. Chem. B, 1998, 5647, 4193-4204.

51 S. Nose, J. Chem. Phys., 1984, 81, 511-519.

52 W. G. Hoover, Phys. Rev. A: At., Mol., Opt. Phys., 1985, 31, 1695-1697.

53 U. Essmann, L. Perera, M. L. Berkowitz, T. Darden, H. Lee and L. G. Pedersen, J. Chem. Phys., 1995, 103, 8577-8593.

54 J.-P. Ryckaert, G. Ciccotti and H. J. Berendsen, J. Comput. Phys., 1977, 23, 327-341.

55 C. Krekeler and L. D. Site, J. Phys.: Condens. Matter, 2007, 4, 192101.

56 W. Kunz, Curr. Opin. Colloid Interface Sci., 2010, 15, 34-39. 57 S. H. Lee and J. C. Rasaiah, J. Phys. Chem., 1996, 100, 1420-1425. 58 T. Lazaridis and M. Paulaitus, J. Phys. Chem., 1992, 96, 3847-3855. 59 J. Hansen and I. McDonald, Theory of Simple Liquids, Academic Press, London, 3rd edn, 2006.
60 Y. Marcus, J. Chem. Soc., Faraday Trans., 1991, 87, 2995-2999.

61 N. T. Southall, K. A. Dill and A. D. J. Haymet, J. Phys. Chem. $B, 2002,106,521-533$.

62 J. N. Israelachvili, Intermolecular and Surface Forces, Academic Press inc. Elsevier, London, 3rd edn, 2011.

63 T. T. Duignan, D. F. Parsons and B. W. Ninham, J. Phys. Chem. B, 2013, 117, 9412-9420.

64 L. Landau and E. Lifshitz, Course of Theoretical Physics: Volume 9: Statistical Physics, Pergamon Press Ltd, Oxford, 1981.

65 M. D. Tissandier, M. D. Tissandier, K. A. Cowen, K. A. Cowen, W. Y. Feng, W. Y. Feng, E. Gundlach, E. Gundlach, M. H. Cohen, M. H. Cohen, A. D. Earhart, A. D. Earhart, J. V. Coe, J. V. Coe, T. R. Tuttle Jr. and T. R. Tuttle Jr., J. Phys. Chem. A, 1998, 102, 7787-7794.

66 C. P. Kelly, C. J. Cramer and D. G. Truhlar, J. Phys. Chem. B, 2006, 110, 16066-16081.

67 E. Lifshitz and L. Pitaevskii, Statistical Physics, Part 2, Theory of the Condensed State, Butterworth-Heinemann, Amsterdam, 1 st edn, 1980.

68 W. F. McDevit and F. A. Long, J. Am. Chem. Soc., 1952, 74, 1773-1777.

69 K. D. Collins and M. W. Washabaugh, Q. Rev. Biophys., 1985, 18, 323-422.

70 K. A. Dill and T. M. Truskett, Annu. Rev. Biophys. Biomol. Struct., 2005, 34, 173-199.

71 M. Geisler, T. Pirzer, C. Ackerschott, S. Lud, J. Garrido, T. Scheibel and T. Hugel, Langmuir, 2007, 29, 1741-1744.

72 C. J. Rizzo, J. Org. Chem., 1992, 57, 6382-6384. 